\title{
Estimación de la subsidencia en el Valle Central (Costa Rica) mediante interferometría diferencial con radar de apertura sintética
}

Estimation of land subsidence using differential synthetic aperture radar interferometry in the Central Valley (Costa Rica)

\author{
Historial del artículo \\ Recibido: \\ 20 de mayo de 2021 \\ Revisado \\ 13 de noviembre de 2021 \\ Aceptado:
}

18 de noviembre de 2021

\author{
Rubén Martínez Barbáchano ${ }^{\mathrm{a}}$ \\ a Universidad de Costa Rica. Correo electrónico: ruben.martinezbarbachano@ucr.ac.cr. ORCID: https://orcid.org/0000-0002- \\ 9176-3112
}

\section{Palabras clave}

Interferometría, radar, Sar, subsidencia, teledetección

\section{Keywords}

Interferometry, radar, remote sensing, Sar, subsidence

\begin{abstract}
Resumen
La interferometría SAR (Synthetic Aperture Radar) es una técnica de teledetección usada para aplicaciones como la generación de modelos digitales del terreno y la monitorización de deformaciones de superficie terrestre. La técnica DInSAR (InSAR diferencial) explota la fase, que es sensible a la topografía del terreno y a los cambios de elevación ocurridos entre dos pasadas del satélite sobre la misma área. Para la obtención de información topográfica se requiere un modelo digital del terreno, y la diferencia de fase permite realizar estimaciones de desplazamiento vertical del terreno con precisiones superiores a 1 centímetro. El presente trabajo permitió conocer la deformación superficial experimentada en las áreas urbanas del Valle Central de Costa Rica, tras la erupción del volcán Turrialba el 1 de agosto de 2020. Para ello se utilizaron las imágenes captadas por el sensor Sentinel 1 de la Agencia Espacial Europea.
\end{abstract}

\section{Abstract}

SAR interferometry Synthetic Aperture Radar is a remote sensing technique used for applications such as digital terrain model generation and monitoring deformations in the earth's surface. DInSAR technique (InSAR differential) exploits the phase, that is sensitive to changes in the topography of the terrain and changes of elevation between two ground tracks over the same area. In order to obtain ground information, a digital terrain model is required, and phase differences allows estimates of ground vertical displacement with better than $1 \mathrm{~cm}$ relative accuracy. The present work allowed to estimate a surface deformation of urban areas of the Central Valley in Costa Rica, after 1st of August 2020 eruption of Turrialba volcano. To that end, several Sentinel-1 images from the European Space Agency have been used. 


\section{Introducción}

La teledetección con radar de apertura sintética (SAR) ha experimentado un renovado impulso en los últimos años, con nuevos sensores y plataformas gratuitas que han permitido la obtención de magnitudes o variables geofísicas de gran utilidad geográfica. La Agencia Espacial Europea (European Space Agency - ESA) tienen en marcha el programa Copérnico, un ambicioso programa espacial de observación de la tierra, que desde 2011 proporciona información de la superficie terrestre y de la atmósfera de forma sistemática, actualizada y de fácil acceso, con el objetivo de mejorar la gestión del medio ambiente, comprender y mitigar los efectos del cambio climático y garantizar la seguridad ciudadana, para lo cual se sirve de los datos procedentes de más de 30 satélites. Entre dichos satélites, cabe mencionar las cinco familias de misiones Sentinel, comenzando por las imágenes radar de Sentinel-1, que desde 2014 nos proporciona observaciones de la superficie de la Tierra en cualquier condición meteorológica, de día o de noche.

Desde el punto de vista del espectro electromagnético, los sistemas RADAR trabajan en el rango de las microondas (es decir, como sensores activos) debido a que la emitancia de los objetos es difícilmente perceptible en esa banda (Chuvieco, 2010).

Entre las técnicas recientes más utilizadas con tecnología radar destaca la interferometría. Esta técnica ha permitido, tanto a escala regional como local, medir deformaciones centimétricas, e incluso milimétricas, de la superficie topográfica bajo ciertas condiciones. En este sentido destacan las aplicaciones en movimientos de masas glaciares (Goldstein et al., 1993) en desplazamientos sísmicos relacionados con fallas activas (Massonet et al., 1993) las deformaciones relacionadas con la actividad volcánica e intrusiones de magma (Sigmudsson, 1999) o el monitoreo de deformaciones de los volcanes a largo plazo (Massonet et al., 1995). También cabe mencionar estudios de subsidencia kárstica (Guerrero, 2009), minera (Herrera et al., 2007), urbana (Cascini et al., 2006), la producida por extracción de aguas subterráneas (Amelung et al., 1999) o la sobreexplotación de acuíferos en áreas urbanas (Tomás et al., 2005).

El funcionamiento de la tecnología RADAR se basa en una señal electromagnética emitida a través de una antena, en sentido lateral a la plataforma que sustenta el sensor. La señal se emite y se dispersa, chocando con las superficies u objetos a los que va dirigida, y la antena radar capta una parte de esas ondas reflejadas, construyendo una imagen de la zona observada.
Los píxeles que conforman una imagen radar representan el coeficiente de retrodispersión de la superficie del terreno, con valores directamente proporcionales a la intensidad de la señal de retorno. A diferencia de los sensores pasivos que trabajan en el espectro óptico, la principal virtud o ventaja que ofrece la tecnología radar es que puede trabajar en cualquier condición atmosférica (Chuvieco, 2010). Ello debido a que el radar trabaja en una longitud de onda comprendida entre $\operatorname{los} 0,1 \mathrm{~cm}$ y $1 \mathrm{~m}$. Aunque la observación de la superficie terrestre en esta longitud de onda entraña dificultades complejas y diferentes a las del espectro óptico, su principal ventaja reside en que es prácticamente transparente a la cobertura nubosa y las influencias atmosféricas, debido a que trabaja en longitudes de onda mayores a $0,1 \mathrm{~mm}$, que es el tamaño habitual de las gotas de agua.

El hecho de que la teledetección con microondas se base en el uso de sensores activos como este, se debe a la escasa emisividad espectral que se da en esta región del espectro, como demuestra la de Planck calculada por Rayleigh-Jeans:

$$
\operatorname{Mn} \lambda=2 \pi \mathrm{kT} / \lambda 4
$$

Donde $\mathrm{Mn} \lambda$ es la emitancia espectral, T es la temperatura en grados Kelvin, y $\lambda$ es la longitud de onda. La anterior expresión demuestra que la emitancia de los objetos es difícilmente detectable en la región de las microondas, razón por la cual la teledetección activa mediante radar es la más extendida en usos y aplicaciones.

Un sensor radar puede configurarse de muy diversas formas, obteniendo así señales de retorno igualmente diversas. De hecho, resulta habitual encontrar sensores radar con distintas bandas de observación, diferentes alturas, diferentes ángulos de observación, diferentes polarizaciones y antenas de tamaño diverso. Por otra parte, la resolución a lo largo y ancho del barrido determina el tamaño del píxel en el terreno (Zhou et al., 2009).

La ecuación fundamental del radar es la siguiente:

$$
\operatorname{Pr}=\operatorname{Pt~G2~} \lambda 2 \sigma /(4 \pi) 3 \text { r4 }
$$

Siendo Pr la potencia de la señal retrodispersada, Pt la potencia emitida por el sensor, $\mathrm{G}$ el factor de ganancia de la antena, $\sigma$ la sección eficaz de retrodispersión y $\mathrm{r}$ la distancia entre el sensor y la cubierta. De la expresión anterior se puede deducir que la señal de retorno es altamente dependiente de la potencia emitida y de la altura 
de observación (Chuvieco, 2010). Tal afirmación puede confirmarse a través de la siguiente ecuación:

$$
\operatorname{Pr}=\operatorname{Pt} A 2 \sigma / 4 \pi \lambda 2 r 4
$$

En la que A representa la superficie de la antena receptora, lo que implica que, a mayor altura de observación del sensor, el tamaño de la antena debería ser mayor para obtener una buena señal. Por ello, a la hora de incorporar sensores radar a plataformas orbitales se requiere de antenas de gran tamaño, que garanticen una gran potencia o elevada ganancia efectiva. Ello plantea un reto logístico o de diseño, dado que para obtener una resolución aceptable los sensores orbitales deberían contar con enormes antenas. Pero este problema puede solventarse en parte con el diámetro de apertura de la antena, representado por $\Phi$ en la siguiente expresión:

$$
\mathbf{R s}=\lambda \mathbf{r} / \boldsymbol{\Phi}
$$

El radar de apertura sintética (SAR) permite simular distintos tamaños de antena mediante el denominado efecto Doppler, consistente en que la resolución del sensor se basa en la obtención de dos señales de retorno de un mismo punto de la superficie terrestre en dos instantes diferentes de la trayectoria del sensor. De este modo, la resolución no depende del tamaño real de la antena, sino de la que se obtendría de una antena de la misma longitud a la distancia existente entre esos dos puntos (Elachi, 1987).

La interferometría se centra en el estudio y explotación de la información proporcionada por las imágenes de fase de las imágenes complejas SAR (Hanssen, 2001). Tanto la técnica de interferometría SAR (InSAR) como la interferometría diferencial (DInSAR) se basan en la explotación contenida en uno o más interferogramas (Klees \& Massonnet, 1998). Concretamente, en obtener la posición de un punto hipotético, en función de la observación (desde un radar SAR) de dicho punto desde dos posiciones distintas. Por tanto, las imágenes SAR contienen en cada píxel información de la amplitud y la fase correspondiente a los elementos del terreno. La amplitud varía en función de las características que la zona de estudio ejerce sobre la señal retrodispersada, mientras que la fase contiene la información de la distancia de la antena al suelo de la zona de estudio (Moreira et al., 2013). El interferograma se forma con dos imágenes SAR, mediante un proceso en el cual cada píxel de la primera imagen se multiplica por el conjugado del mismo píxel de la segunda imagen, obteniéndose una nueva imagen en la que los valores que contiene son la multiplicación de los valores de la amplitud y la diferencia de fase en cada uno de los píxeles de una imagen respecto de la otra.

En la señal retrodispersada que recibe el sensor, además de la distancia al punto y las características de la superficie, influyen otros factores como el ruido inherente al sistema de medida o las condiciones atmosféricas, que influyen en la calidad del producto obtenido (Moreira et al., 2013). Tal y como ha sido expuesto, por tanto, se puede determinar la posición de un punto mediante el cálculo de la variación de distancia que existe al observar el punto desde dos posiciones distintas del satélite, pudiendo ocurrir también que la variación del valor de distancia se deba a una variación en la posición del punto (figura 1).

Por todo ello, para poder aislar y cuantificar el valor de la diferencia de fase correspondiente al desplazamiento del punto nació la interferometría diferencial SAR conocida como DinSAR. Su rasgo más característico es que elimina los efectos topográficos incluidos en la fase del punto utilizando un modelo digital de elevaciones, generando lo que se conoce como un interferograma diferencial. Al suprimir el componente topográfico del valor de la fase, quedarían otros factores que contribuyen al desplazamiento sufrido por el punto; se trata de las condiciones atmosféricas y el ruido de fase debido a la decorrelación temporal y variaciones del medio de propagación. (Moreira et al., 2013).

En cuanto a la capacidad y límites de esta técnica en la identificación de procesos volcánico-sísmicos como el que nos ocupa, existe un límite interferométrico que viene dado por la expresión:

\section{$2 \mathrm{~L}(\operatorname{sen} \varphi 1-\operatorname{sen} \varphi 2)<\lambda$}

Donde L es la longitud del píxel perpendicular a la trayectoria, $\varphi 1$ y $\varphi 2$ son los ángulos de incidencia (desde la vertical local) en la primera y segunda imagen comparada y $\lambda$ es la longitud de onda de la radiación emitida.

\section{Materiales y métodos}

La cordillera volcánica central de Costa Rica constituye un eje montañoso de $2.474 \mathrm{~km}^{2}$ orientado de noroeste a sureste a lo largo de $80 \mathrm{~km}$, ubicada entre las coordenadas geográficas: 9 $9^{\circ} 49^{\prime} 4$ '” $\mathrm{N} / 84^{\circ} 11^{\prime} 4^{\prime \prime} \mathrm{O}$ y $10^{\circ} 21^{\prime} 20^{\prime \prime} \mathrm{N}$ $/ 85^{\circ} 3^{\prime} 40^{\prime \prime} \mathrm{O}$. Se trata de un sistema montañoso del Cuaternario caracterizado por la existencia de dos vertientes muy contrastadas desde el punto de vista de modelado morfoclimático; la vertiente norte de barlovento, muy erosionada con grandes barrancos y deslizamientos por 


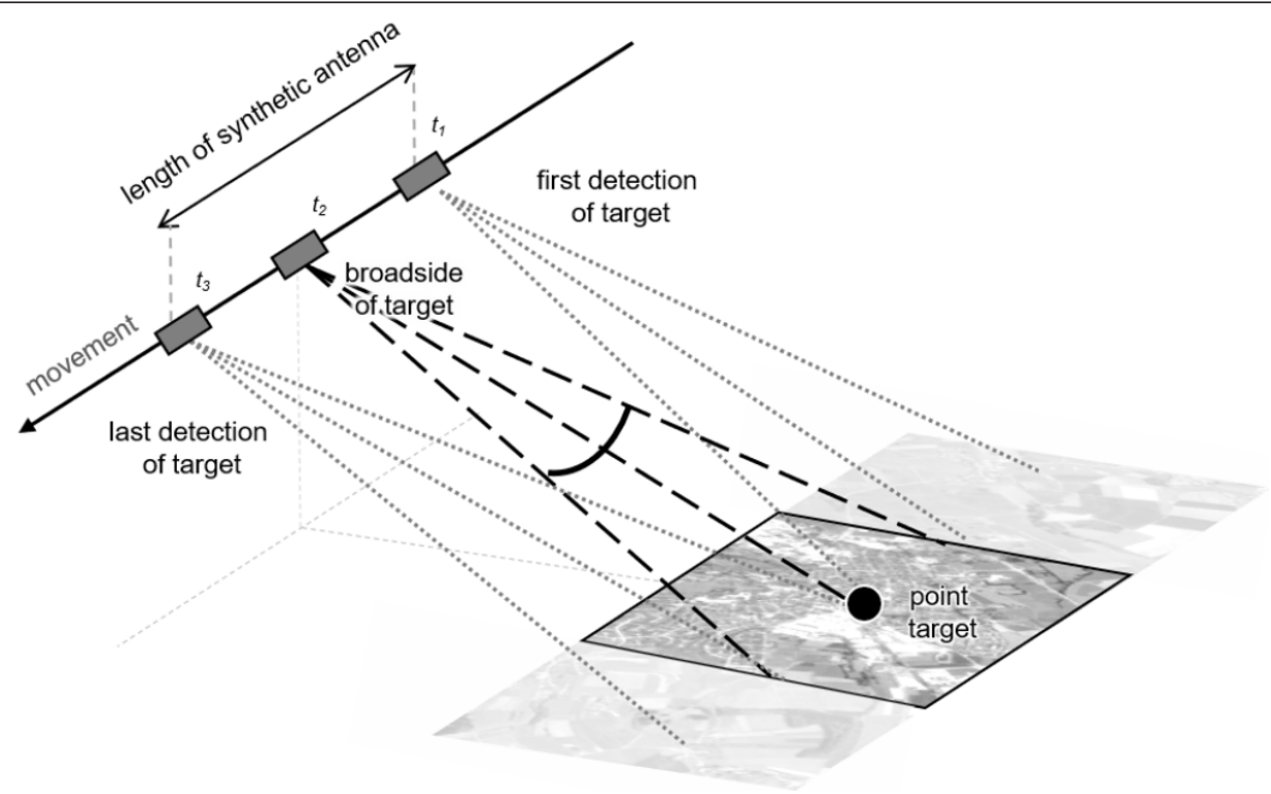

Figura 1. Trayectoria y adquisición de un sistema radar. Fuente: Braun (2019)..

la acción de las altas precipitaciones y la vertiente sur de sotavento, donde las coladas y lahares están cubierta por espesas capas de ceniza y se encuentra menos meteorizada, al estar más protegida de los vientos alisios de noreste (Bergoeing, 1998).

En el límite sur de la cordillera volcánica central se encuentra el Valle Central o depresión tectónica central, delimitada por los valles de los ríos Grande y Virilla. Hacia el este, los cerros de Ochomogo delimitan el Valle Central en su sector occidental, donde se sitúan las ciudades de San José, Heredia y Alajuela y el sector oriental, donde se asientan las ciudades de Cartago y Turrialba (Bergoeing, 2014).

Desde un punto de vista geomorfológico, el sur del área de estudio (donde se obtuvieron las mayores deformaciones) está recorrido por la falla Agua Caliente, que inicia en la Loma Salitral y continua hasta el sur de Paraíso de Cartago (figura 6). Se trata de una falla sinuosa con rumbo variable noroeste/sureste, entre la Loma Salitral (sur de Desamparados) y el sur de la ciudad de Cartago. Se han identificado depósitos del Cuaternario Superior cortados por fallas inversas asociadas a esta falla (Montero et al., 2006) que además tiene un movimiento predominante siniestral con una zona transtensiva entre Quebrada Honda y Bermejo, al sur de Cartago. En el extremo occidental de la falla se encuentra la mencionada Loma Salitral, una loma aislada cuya extraña ubicación se relaciona por el desplazamiento siniestral a lo largo de la falla Agua Caliente, que atraviesa la loma.

De acuerdo con Solano y Villalobos (2001) el Valle Central se ve afectado por condiciones del Pacífico (zona de convergencia intertropical y vientos ecuatoriales) así como por la influencia del Caribe (Alisios del noreste). Ello explicaría el clima seco con marcada influencia del Pacífico en las partes más bajas del valle occidental (La Garita, Guácima) un clima templado en San José, Heredia y Cartago, y un clima más lluvioso y frío en las zonas altas, con un rango de precipitaciones que oscila entre los 1700 a los $2820 \mathrm{~mm}$ anuales, y temperaturas variables entre $10-21{ }^{\circ} \mathrm{C}$ con una elevada humedad relativa, superior al $75 \%$ buena parte del año.

Todo el procesamiento de imágenes que sustenta la presente metodología se realizó con el software de código abierto SNAP, desarrollado por la Agencia Espacial Europea. Dado que la técnica DInSAR (InSAR diferencial) se basa en los cambios de elevación ocurridos entre dos barridos del satélite sobre un mismo lugar, el primer paso consistió en escoger las imágenes inmediatamente anterior y posterior a la fecha de erupción del volcán Turrialba, el 1 de agosto de 2020. Las imágenes Sentinel 1 están disponibles para libre descarga en el sitio web Copernicus Open Access 
Tabla 1

Precios por metro cuadrado de terreno limpio y con servicios. US\$ a valores corrientes. Quebrada de Humahuaca. Años 2004, 2011, 2016 y 2018.

\begin{tabular}{|c|c|}
\hline Tipo de procesamiento & Herramientas de software \\
\hline 1. Extracción de franjas útiles. & 1. Herramienta Split. \\
\hline \multirow{2}{*}{ 2. Corregistro de imágenes. } & 2. Back Geocoding. \\
\hline & 3. Corrección orbital. \\
\hline $\begin{array}{l}\text { 3. Creación del interferograma y } \\
\text { estimación de la coherencia. }\end{array}$ & 2. Interferogram Formation. \\
\hline 4. Eliminación de bandeado. & 5. Herramienta TOPS Deburst. \\
\hline 6. Reducción del ruido de la imagen. & 6. Filtro Goldstein. \\
\hline $\begin{array}{l}\text { 7. Desenrollado de franjas de la } \\
\text { imagen de fase. }\end{array}$ & 7. Plugin SNAPHU Unwrapping. \\
\hline $\begin{array}{l}\text { 8. Conversión de la fase a } \\
\text { desplazamiento. }\end{array}$ & $\begin{array}{l}\text { 8. Herramienta Phase to } \\
\text { Displacement. }\end{array}$ \\
\hline 9. Corrección geométrica. & $\begin{array}{l}\text { 9. Range Doppler Terrain } \\
\text { Correction. }\end{array}$ \\
\hline \multirow{2}{*}{$\begin{array}{l}\text { 10. Generación de productos } \\
\text { finales. }\end{array}$} & $\begin{array}{l}\text { 10.1 Aplicación de máscara de } \\
\text { coherencia. }\end{array}$ \\
\hline & $\begin{array}{l}\text { 10.2 Exportar a formatos } \\
\text { cartográficos. }\end{array}$ \\
\hline
\end{tabular}

Fuente: Elaboración propia a partir de la guía para usuarios de Sentinel $1 \mathrm{~A}$ de la Agencia Espacial Europea. https://sentinel.esa.int/web/sentinel/ user-guides/sentinel-1-sar/product-types-processing-levels/level-1.

$\mathrm{Hub}^{1}$ (anteriormente conocido como Sentinels Scientific Data Hub) que proporciona acceso completo, gratuito $\mathrm{y}$ abierto a los productos de usuario de las misiones Sentinel-1, Sentinel-2, Sentinel-3 y Sentinel-5P.

Las imágenes utilizadas correspondían al 23 de julio y al 4 de agosto de 2020, del satélite Sentinel 1A, nivel de producto SLC (Single-Look Complex) en modo Interferometric Wide Swath (IW) que implica un barrido de 3 franjas paralelas dirigiendo la antena a tres posiciones across-track (a lo ancho del barrido) cuyo resultado es un ancho de escena de $250 \mathrm{~km}$ con una resolución de 5 x 20 $\mathrm{m}$. Las imágenes seleccionadas para el presente estudio fueron las más cercanas al evento eruptivo del volcán, por lo que se descartaron otras imágenes Sentinel 1 de pasada ascendente, más alejadas de la fecha del evento (25 días) $\mathrm{y}$, por tanto, más propensas a reflejar en la rampa de fase eventos o factores ajenos a la erupción del volcán Turrialba el 1 de agosto de 2020. Se procesaron posteriormente los interferogramas disponibles correspondientes al resto de meses del año 2020 (desde enero hasta diciembre) con objeto de comprobar discordancias o patrones identificables a lo largo del año.
El flujo de procesamiento de las imágenes radar en interferometría se divide en varias fases, tal y como muestra la tabla 1.

Como paso previo al procesamiento, se realizó un subset consistente en seleccionar únicamente las franjas de barrido de ambas imágenes que abarcaban el área de estudio, mediante la herramienta S-1 TOPS Split. Una vez realizado el subset de franjas de barrido, el siguiente paso fue el corregistro de imágenes obtenidas en el paso anterior, pero antes es necesario aplicar en las imágenes obtenidas anteriormente los archivos con la información detallada de las órbitas, mediante la herramienta de SNAP "Apply Orbit File".

Una vez aplicada la información de la trayectoria orbital en las imágenes, se procede a aplicar el corregistro. Se trata de un proceso consistente en posicionar los píxeles de una imagen (la del 23 de julio) con los mismos píxeles correspondientes otra imagen (la del 4 de agosto) para poder generar correctamente un interferograma. Para ello se selecciona una imagen (normalmente la más antigua) como imagen de referencia y la otra imagen (la más reciente) se selecciona como subordinada. La herramienta que el software SNAP proporciona para ello se denomina "S-1 TOPS Corregistration".

Para que este proceso se realice con éxito se requiere la información precisa de las órbitas del satélite y un modelo digital de elevación del área abarcada. SNAP permite descargar automáticamente de internet tanto las órbitas (vistas en el paso anterior) como la información topográfica (modelo digital de elevación). Para el presente trabajo se utilizó el modelo digital SRTM 1 sec HGT, un producto de la NASA (Shuttle Radar Topography Mission) generado a partir de datos radar SAR de banda $\mathrm{C}$ y X con una resolución de 3 arcosegundos, es decir, 30 metros. El método de remuestreo para alinear los puntos de la imagen subordinada con los de la imagen de referencia fue el de interpolación bilineal, recomendada para conjuntos de datos continuos sin límites definidos. Cabe mencionar por último que la presente técnica y el software solo permiten corregistrar una subfranja (IW2) con una única polarización (vertical-vertical).

El siguiente paso que se realizó fue la formación del interferograma. El interferograma (figura 2) se genera al multiplicar la imagen de referencia con la imagen subordinada, lo que produce tres nuevas imágenes complejas; de amplitud, de fase y de coherencia interferométrica.

${ }^{1}$ Ver https://scihub.copernicus.eu/dhus/\#/home 


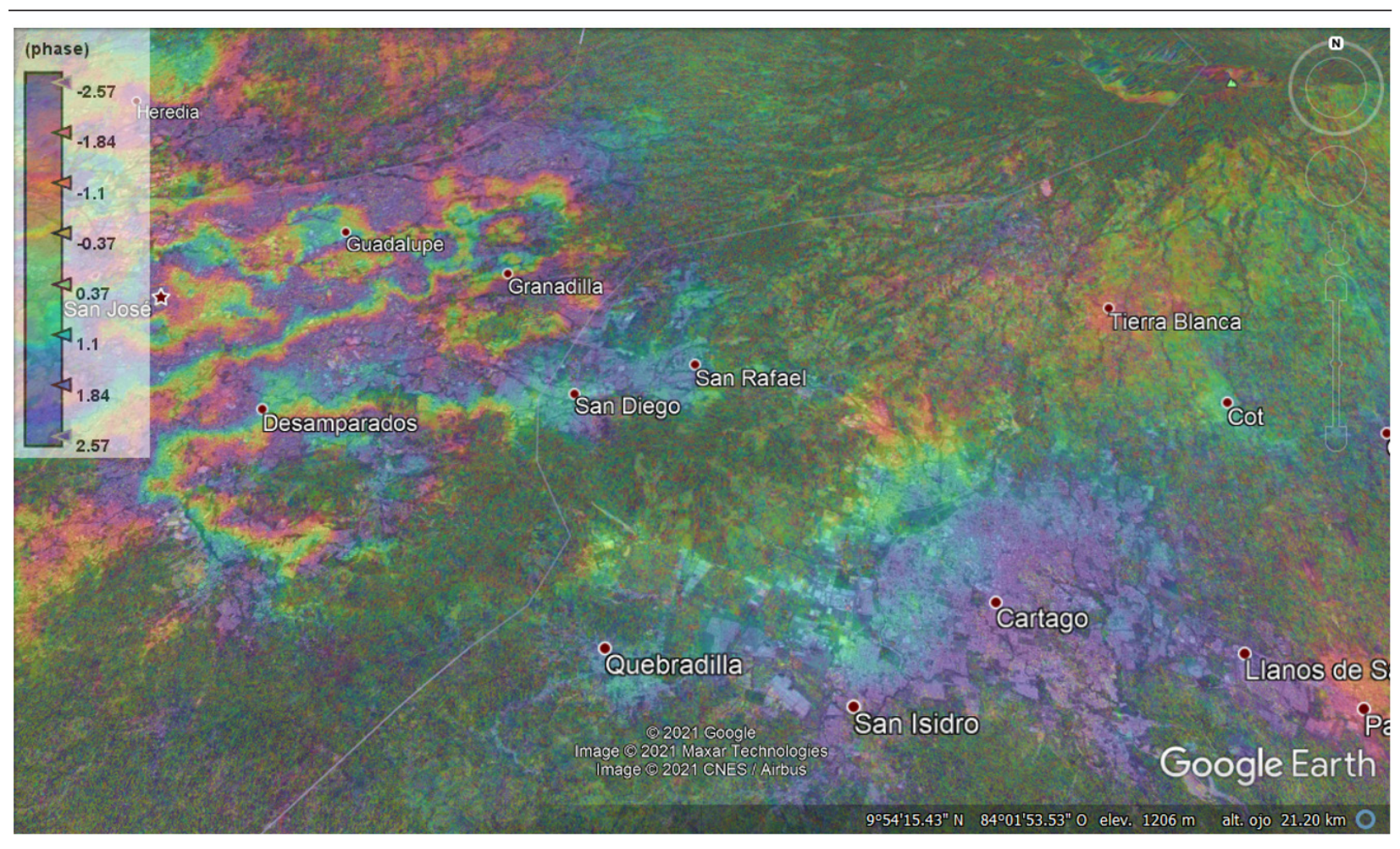

Figura 2. Interferograma del Valle Central (Costa Rica) entre el 23 de julio y el 4 de agosto. Fuente: Elaboración propia a partir de datos Sentinel 1 (Agencia Espacial Europea).

Una vez generados, los interferogramas necesitan un pre-procesado que corresponde fundamentalmente con la eliminación del ruido (speckle) y un posterior procesamiento de la imagen mediante un protocolo previamente establecido (Richards et al., 2010). Concretamente, y debido a la forma de adquisición de los datos Sentinel 1, las imágenes pueden presentar subfranjas dentro de la cuales se producen solapes. Para ello, el software SNAP proporciona la herramienta "S1 TOP Deburst", que une las distintas ráfagas teniendo en cuenta el solape, por lo que desaparecen las líneas intermedias, quedando una imagen continua.

El paso siguiente consistió en la creación del interferograma diferencial, eliminando el efecto de la topografía de la zona sirviéndose de un modelo digital de elevaciones (se utilizó siempre el modelo digital SRTM 1 HGT de 3 arcosegundos). La herramienta que lo permite en SNAP es "Topographic Phase Removal":

Algunos de los problemas asociados a la generación de interferogramas de fase es el ruido por problemas de decorrelación geométrica, retrodispersión y otros errores de procesamiento (Braun \& Veci, 2015). Y aunque la información de fase en áreas decorreladas no puede ser restituida en tales casos, la calidad de las franjas existentes en el interferograma puede ser incrementada aplicando filtros como el de Goldstein, basado en la función transformada rápida de Fourier (FFT), un algoritmo que reduce el tiempo de cálculo, con resultados óptimos en la mayor parte de la imagen tratada, y donde no es así, el usuario es advertido por los cortes (o cambios de color) de las franjas (Goldstein et al., 1988).

De la figura 2 podía deducirse fácilmente que el ruido de las imágenes interferométricas diferenciales dificulta su interpretación, y entre los filtros disponibles en el software SNAP, destaca el citado filtro de fase de Goldstein. El interferograma presenta, no obstante, algunos inconvenientes aún. El principal es que para poder establecer una correlación entre la fase interferométrica y la altura topográfica, hay que desenvolver el archivo correspondiente a la fase. La razón es que los valores de fase están ceñidos a un intervalo de 2 $\pi$, y al desenvolver la imagen se integran la diferencia de fase entre píxeles vecinos. Por tanto, la imagen desenvuelta puede ser interpretada como la altura/desplazamiento entre píxeles de dos imágenes (Braun \& Veci, 2015). La herramienta que permitió realizar este proceso en el software de SNAP es un plugin denominado "SNAPHU 

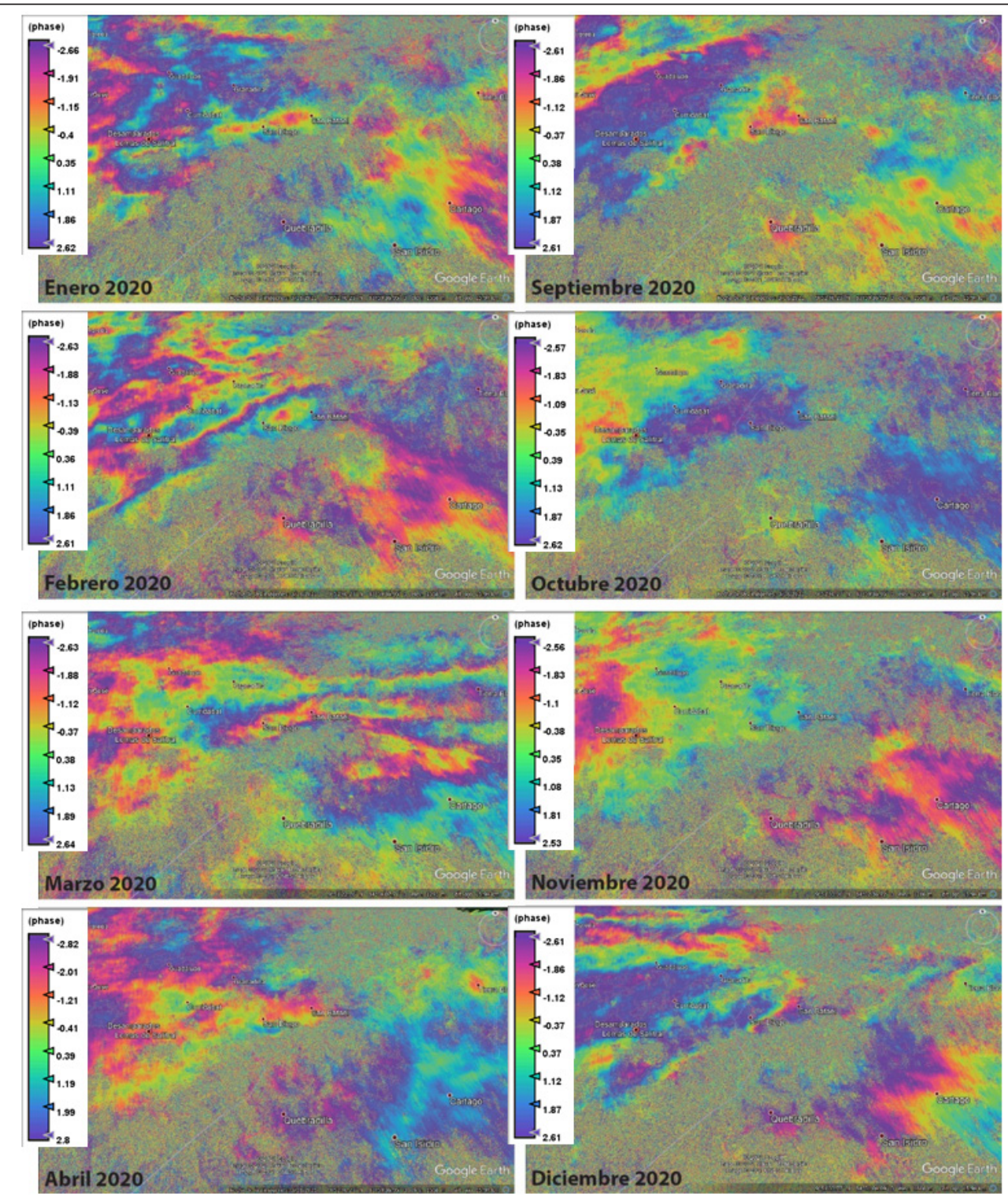

Figura 3. interferogramas procesados del Valle Central (Costa Rica) en el año 2020. Fuente: elaboración propia a partir de datos Sentinel 1 (Agencia Espacial Europea).

Phase Unwrapping". Una vez obtenido el raster de fase desenvuelto, para poder establecer relaciones paramétricas hay que convertir las unidades de la imagen de radianes en desplazamientos cuantificables. La herramienta de SNAP que permite realizar este proceso es "Phase to Displacement", y el producto generado es una capa de tipo raster en la que cada píxel representa los valores de desplazamiento.

El último paso en el procesamiento interferométrico sería la corrección geométrica. La herramienta de SNAP "Range Doppler Terrain Correction" permite crear un interferograma diferencial mediante la sustracción de la topografía de la zona (como "foreshortening" o sombras topográficas) utilizando un modelo digital de elevaciones, en este caso el SRTM 1 HGT de 3 arcosegundos utilizado anteriormente. El resultado es un producto cartográfico proyectado y georreferenciado. Finalmente, se exportaron el raster de desplazamiento y de coherencia, geométricamente corregidos, a formato KMZ para su visualización en Google Earth.

Con objeto de verificar si las deformaciones obtenidas entre el 23 de julio y el 4 de agosto de 2020 fueron un fenómeno aislado o se trataba de un patrón recurrente, se repitió el proceso para todas las imágenes disponibles del año 2020 (figura 3). 


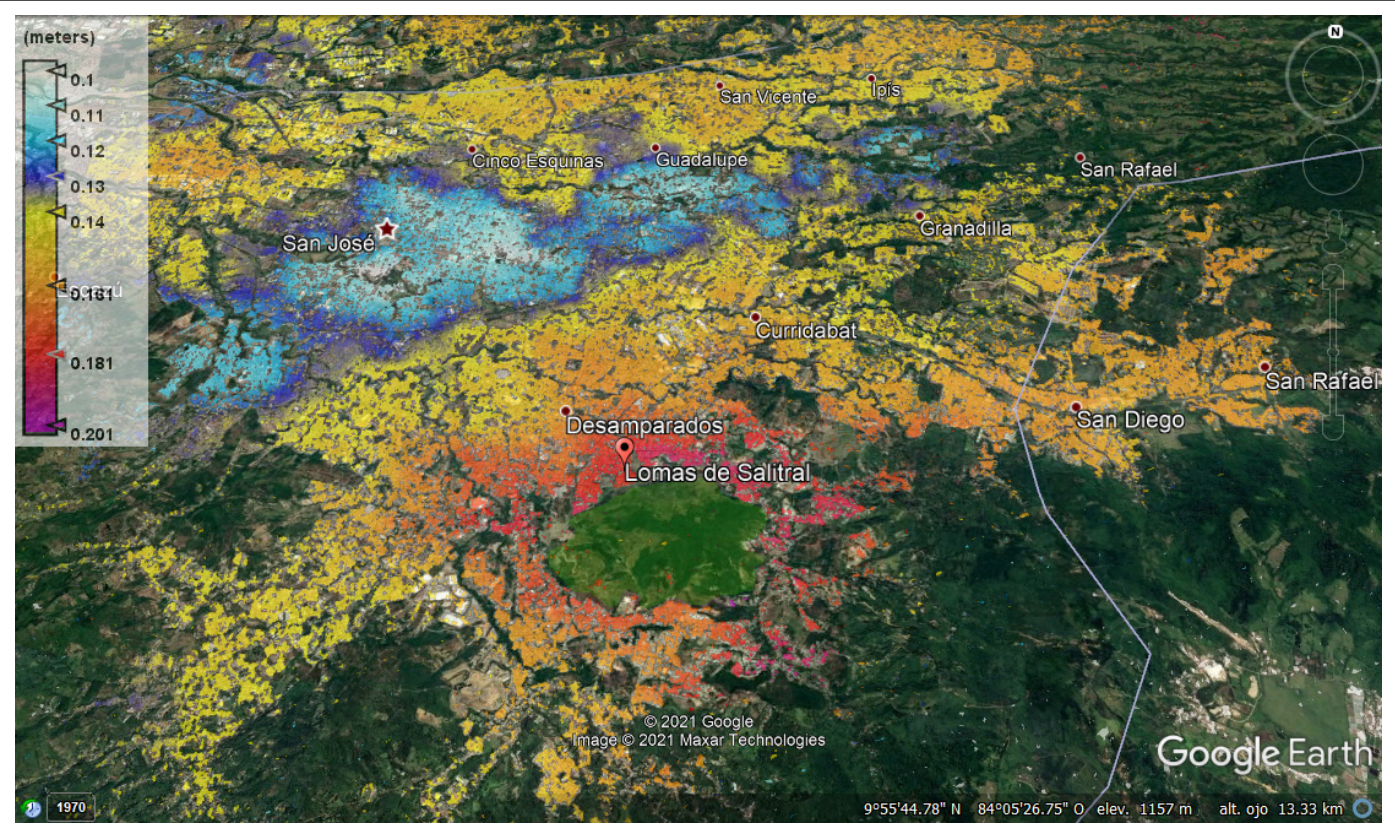

Figura 4. Desplazamiento vertical en San José (Costa Rica) entre el 23 de julio y el 4 de agosto. Fuente: Elaboración propia a partir de datos Sentinel 1 (Agencia Espacial Europea).

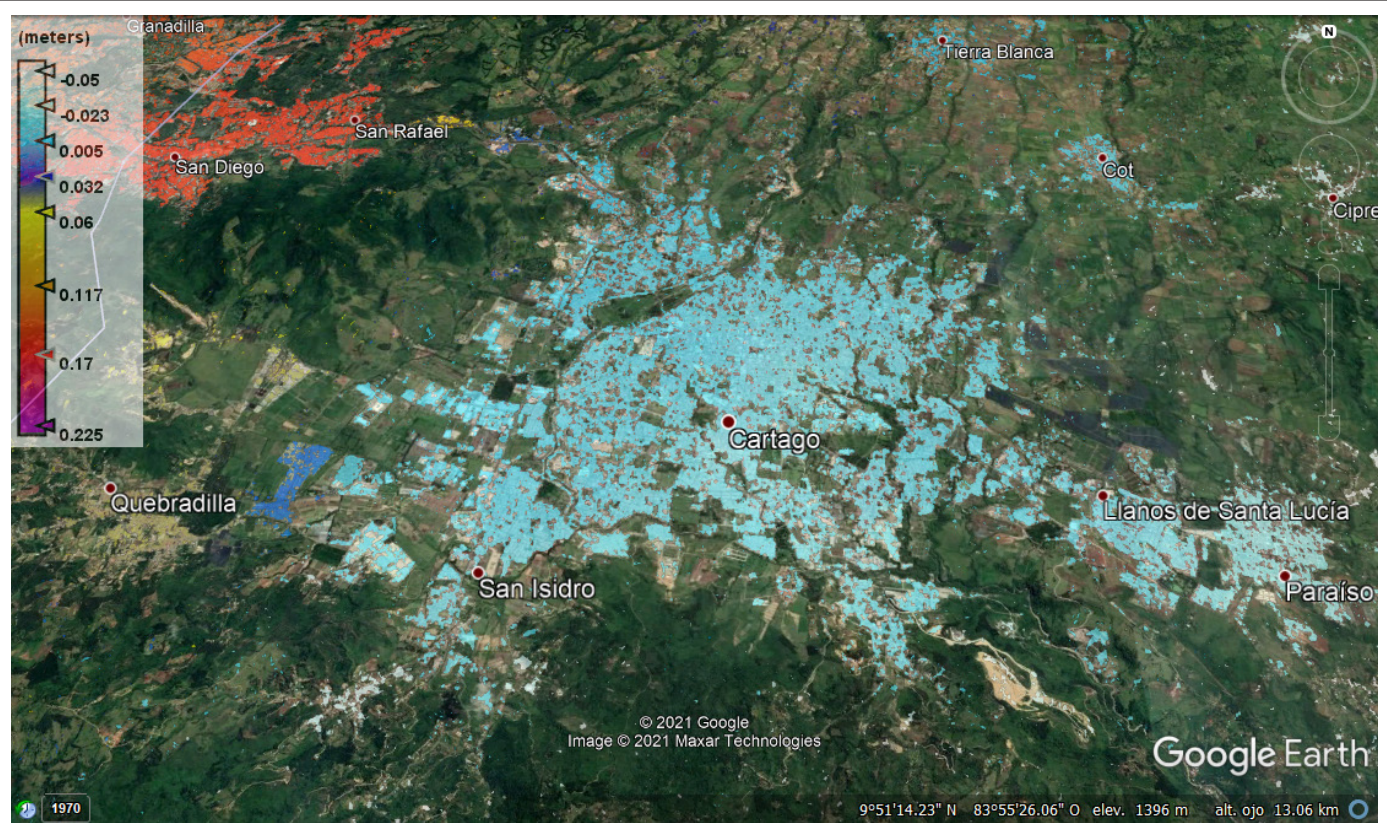

Figura 5. Desplazamiento vertical en Cartago (Costa Rica) entre el 23 de julio y el 4 de agosto. Fuente: Elaboración propia a partir de datos Sentinel 1 (Agencia Espacial Europea).

\section{Resultados}

El procesamiento interferométrico de las imágenes de fase y coherencia dieron como resultado un levantamiento generalizado del terreno en el sur de San José (figura 4) y una leve subsidencia en la ciudad de Cartago y alrededores (figura 5).
Los valores máximos se alcanzaron alrededor de la Loma Salitral, al sur de Desamparados (más de $15 \mathrm{~cm}$ ), mientras en la ciudad de Cartago, la deformación fue descendente o subsidente (figura 5) con valores de 0 a -2 centímetros (dentro del conjunto urbano comprendido entre Cartago, El Tejar, San Isidro, Cot, Llanos de Santa Lucía y Paraíso). 


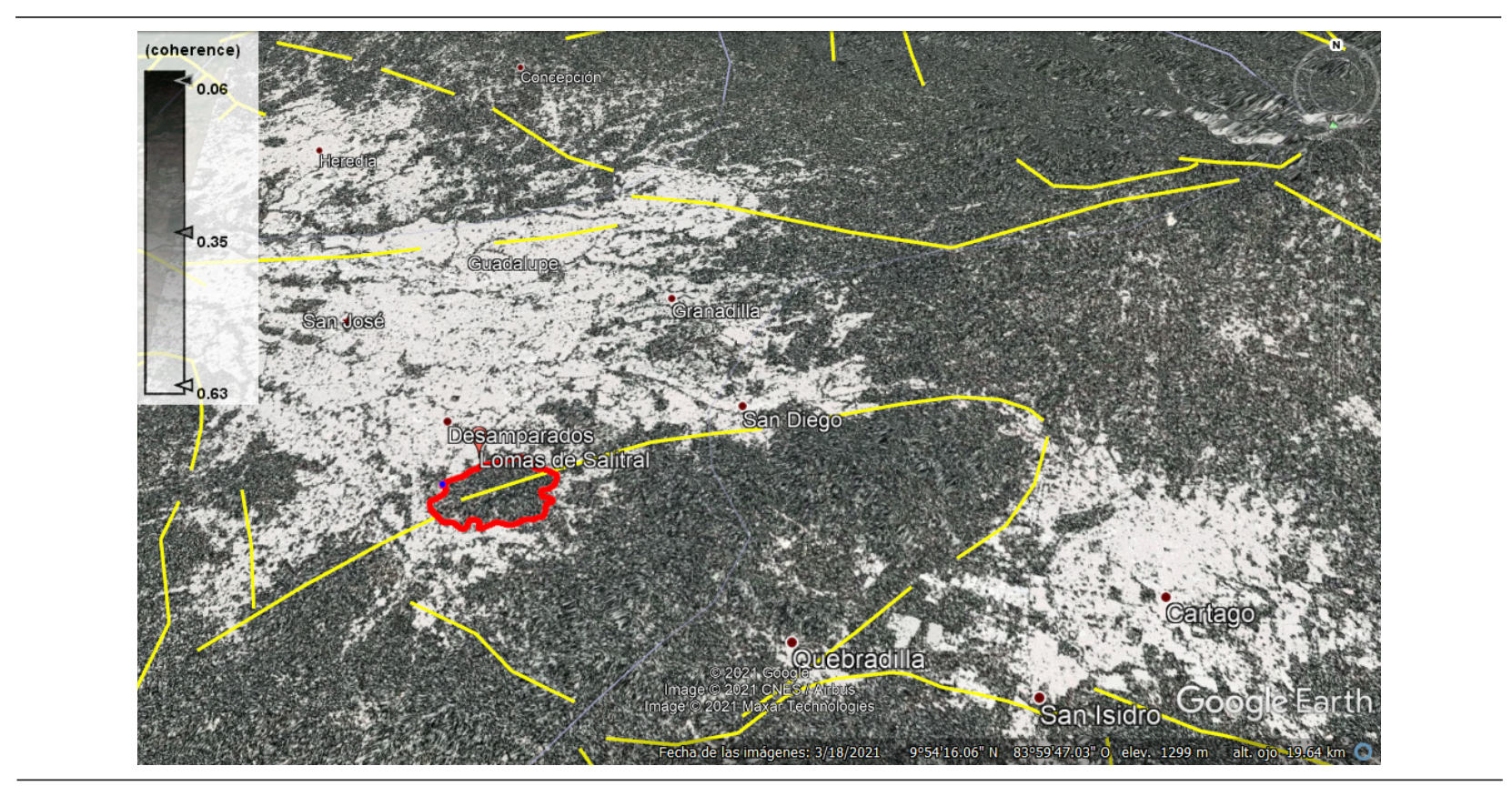

Figura 4. Coherencia interferométrica del área de estudio entre el 23 de julio y el 4 de agosto.

Fuente: Elaboración propia a partir de datos Sentinel 1 (Agencia Espacial Europea).

Los valores de deformación obtenidos para el resto de los meses del año 2020 fueron próximos a cero en todo el Valle Central, de acuerdo a los interferogramas procesados (figura 3) con valores superiores a 0,6 en las imágenes de coherencia para el área de estudio.

\section{Discusión}

La fiabilidad de los datos de deformación obtenidos viene respaldada por la imagen de coherencia (figura 6) que indica valores superiores a 0,6 en toda el área urbana del Valle Central. Cabe recordar en este sentido que la coherencia $(\gamma)$ es un parámetro cuya magnitud está relacionada con la calidad conseguida en la estimación de la fase interferométrica, y varía entre 0 (baja calidad) y 1 (alta calidad) (Herrera et al., 2009). Normalmente se obtienen altos valores de coherencia temporal en superficies con poca vegetación, tal y como sucede en el Gran Área Metropolitana (San José, Alajuela, Cartago y Heredia). Es importante, en todo caso, realizar las observaciones de imágenes SAR simultáneamente, o tan cercanas en el tiempo como sea posible (Siqueira et al., 2019). Otro aspecto para considerar son las condiciones meteorológicas, ya que los cambios enmascaran u ocultan la parte de la fase interferométrica debida a los movimientos del terreno (Herrera et al., 2009). En este sentido el período transcurrido entre la adquisición de las dos imágenes se caracterizó por una canícula más o menos estable desde el 20 de julio hasta la primera semana de agosto (Instituto Meteorológico de Costa Rica, 2020).

\section{Conclusión}

El presente estudio permitió estimar unas deformaciones, entre julio y agosto de 2020, coincidentes con la cierta actividad volcánica del volcán Turrialba y con el sistema de fallas locales. Cabe mencionar, no obstante, que no se puede establecer una relación directa entre las deformaciones estimadas en el Valle Central y la erupción del 1 agosto del volcán Turrialba. Se requeriría de un estudio más profundo para establecer correlaciones entre actividad sísmica, volcánica y deformación de la superficie en el Valle Central.

Dado que la gran mayoría de temblores y enjambres sísmicos que han ocurrido entre 1984 y 2005 tuvieron su epicentro en la zona de cambio de rumbo de la falla Agua Caliente (Montero et al., 2006) convendría profundizar en el seguimiento y la influencia que esta falla podría tener con el levantamiento de la Loma Salitral observado entre el 23 de julio y el 4 de agosto de 2020. Dichas deformaciones son ascendentes (levantamiento) y presentan valores de más de $15 \mathrm{~cm}$ en Guatuso de Patarrá o San Lorenzo de Desamparados. Con posterioridad al evento (diciembre de 2020) el Instituto de Investigación y Observatorio Vulcanológico de Costa Rica (OVSICORI) registró un total de 40 temblores con un rango de magnitud de 0 a 3 en el sur de San José y Desamparados.

Por último, conviene recordar que las técnicas de interferometría diferencial con radar de apertura sintética 
se han desarrollado y validado en los últimos años en estudios de deslizamientos y movimientos sísmicos, pero su aplicación en la mejora de los modelos de predicción de esos eventos se encuentra aún en una fase incipiente.

\section{Referencias}

Amelung, F., Galloway, D. L., Bell, J. W., Zebker, H. A., \& Laczniak, R. J. (1999). Sensing the ups and downs of Las Vegas: InSAR reveals structural control of land subsidence and aquifer-system deformation. Geology, 27(6), 483-486. https://doi. org/10.1130/0091-7613(1999)027<0483:STUADO> 2.3. $\mathrm{CO} ; 2$

Bergoeing, J.P. (1998). Geomorfología de Costa Rica. Instituto Geográfico Nacional.

Bergoeing, J.P. (2014). Geomorfología Regional de Costa Rica. Gerüst creaciones.

Braun, A. (2019). Radar satellite imagery for humanitarian response. Bridging the gap between technology and application [Disertación, Universität Tübingen]. https://hdl.handle.net/10900/91317

Braun, A., \& Veci, L. (2015). TOPS Interferometry Tutorial. Sentinel-1 Toolbox

Veci, L. (2015). TOPS Interferometry Tutorial. Sentinel-1 Toolbox.

Cascini, L., Ferlisi, S., Fornaro, G., Lanari, R., Peduto, D. \& Zeni, G.. (2006). Subsidence monitoring in Sarno urban area via multi-temporal DInSAR technique. International Journal of Remote Sensing - INT J REMOTE SENS, 27, 1709-1716. https://doi. org/10.1080/01431160500296024

Chuvieco, E. (2010). Teledetección Ambiental. La Observación de la Tierra Desde el Espacio. Editorial Ariel.

Elachi, C. (1987). Introduction to the Physics and Techniques of Remote Sensing. Geological Magazine, 125(4), 467-467. https://doi.org/10.1017/ S0016756800013133

Goldstein, R.M., Engclhardt, H., Kamb., I.L., \& Frolich, R.M. (1993). Satellite Radar Interfcromctry for Monitoring Icc Sheet Motion: Application to an Antarctic Icc Stream", Science, 262, 1525- 1530.
Goldstein, R. M., Zebker, H. A., \& Werner, C. L. (1988). Satellite radar interferometry: Two-dimensional phase unwrapping. Radio science, 23(4), 713-720. https://doi.org/10.1029/RS023i004p00713

Guerrero, J. (2009). Dinámica fluvial y riesgos naturales derivados de la subsidencia kárstica en los valles del Huerva y Ebro [Tesis doctoral, Universidad de Zaragoza].

Hanssen, R. (2001). Radar Interferometry. Academic Publishers 2001. Data Interpretation and Error Analysis. https://doi.org/10.1007/0-306-47633-9

Herrera, G., Tomás, R., Lopez-Sanchez, J.M., Delgado, J., Mallorquí, J.J., Duque, S., \& Mulas, J. (2007). Advanced DInSAR analysis on mining areas: La Union case study (Murcia, SE Spain). Engineering Geology, 90(3-4), 148-159. https://doi.org/10.1016/j. enggeo.2007.01.001

Herrera, G., Tomás, R., López-Sánchez, J., Monserrat, O., Cooksley, G. \& Mulas, J. (2009). Sistemas radar aplicados a la investigación de subsidencia $y$ movimientos de ladera. http://hdl.handle. net/10045/21682

Instituto Meteorológico de Costa Rica. (2020). Boletín julio-septiembre. https://www.imn.ac.cr/ documents/10179/29808/PerspClimCR_202006. pdf/6be1d5b4-1f2a-4c57-9e6a-bfdd01aa6b59

Klees, R., \& Massonnet, D. (1998). Deformation measurements using SAR interferometry: potential and limitations. Geologie en Mijnbouw, 77(2), 161176. http://dx.doi.org/10.1023/A:1003594502801

Massonnet, D., Briole, P., \& Arnaud, A. (1995). Deflation of Mount Etna monitored by spaceborne radar interferometry. Nature, 375, 567-570. https:// doi.org/10.1038/375567a0

Massonnet, D., \& Feigl, K. L., (1998). Radar interferometry and its application to changes in the Earth's surface. Reviews of Geophysics, 36(4), 441500. https://doi.org/10.1029/97rg03139

Massonnet, D., Rossi, M., Carmona-Moreno, C., Adragna, F., Peltzer, G., Feigl, K., \& Rabaute, T. (1993). The displacement field of the Landers earthquake mapped by Radar interferometry. Nature, 364, 138-142. https://doi.org/10.1038/364138a0 
Montero, W., Barahona, M., Rojas, W., \& Taylor, M. (2006). Los sistemas de falla Agua Caliente y Río Azul y relevos compresivos asociados, Valle Central de Costa Rica. Revista Geológica De América Central, 33. https://doi.org/10.15517/RGAC.V0I33.4233

Moreira, A., Prats-Iraola, P., Younis, M., Krieger, G., Hajnsek, I., \& Papathanassiou, K.P. (2013). A tutorial on synthetic aperture radar. IEEE Geoscience and Remote Sensing Magazine, 1(1), 6-43. https://doi. org/10.1109/MGRS.2013.2248301

Richards, M., Scheer, J.A. \& Holm, W.A. (2010). Principles of modern radar: Basic principles. https:// doi.org/10.1049/SBRA021E

Sigmundsson, F., Durand, P., \& Massonnet D., (1999). Opening of an eruptive fissure and seaward displacement at Piton de la Fournaise volcano measured by RADARSAT satellite radar interferometry. Geophysical Research Letters, 26(5), 533-536. https://doi.org/10.1029/1999GL900055

Siqueira, P., Flores, A., Herndon, K., Thapa, R., \& Cherrington, E. (2019). The SAR Handbook: Comprehensive Methodologies for Forest Monitoring and Biomass Estimation. https://doi.org/10.25966/ nr2c-s697

Solano, J. \& Villalobos, R. (2001). Aspectos fisiográficos aplicados a un bosquejo de regionalización geográficoclimático de Costa Rica. Tópicos de Meteorología y Oceanografia, 8(1), 26-39. https://docplayer. es/60564030-Aspectos-fisiograficos-aplicados-a-unbosquejo-de-regionalizacion-geografico-climaticode-costa-rica.html

Tomás, R., Márquez, Y., Lopez-Sanchez, J., Delgado, J., Blanco, P., Mallorqui, J., Martínez, M., Herrera, G. \& Mulas, J. (2005). Mapping ground subsidence induced by aquifer overexploitation using advanced Differential SAR Interferometry: Vega Media of the Segura River (SE Spain) case study. Remote Sensing of Environment, 98(2-3), 269-283. https://doi. org/10.1016/j.rse.2005.08.003

Zhou, X., Chang, N. B., \& Li, S. (2009). Applications of SAR Interferometry in Earth and Environmental Science Research. Sensors, 9(3), 1876-1912. https:// doi.org/10.3390/s90301876 